\title{
Máquina de inferência baseada na teoria bayesiana para identificar os estados de ânimo do aluno em um ambiente virtual de aprendizagem
}

\author{
Magalí T. Longhi, Patricia A. Behar, Magda Bercht \\ Programa de Pós-Graduação em Informática na Educação \\ Universidade Federal do Rio Grande do Sul (UFRGS) \\ Caixa Postal 5071 - 90.041-970 - Porto Alegre - RS - Brasil \\ mlonghi@cesup.ufrgs.br, behareterra.com.br, berchteinf.ufrgs.br
}

\begin{abstract}
Resumo. Este artigo apresenta a avaliação de um processo de inferência de estados de ânimo (animado, desanimado, satisfeito ou insatisfeito) baseado em redes bayesianas. O principal objetivo da pesquisa é dotar ambientes virtuais de aprendizagem (AVAs) com uma ferramenta apta a reconhecer a motivação do aluno. A máquina de inferência proposta considera os traços de personalidade, os fatores motivacionais obtidos através dos padrões de comportamento e a subjetividade afetiva identificada em textos disponibilizados nas funcionalidades de comunicação dos AVAs.
\end{abstract}

Palavras-chave. Modelagem afetiva de aluno, redes bayesianas, ambientes virtuais de aprendizagem

Abstract. This article presents studies and evaluation of an inference process of the moods (cheerful, cheerless, satisfied and dissatisfied) based on Bayesian networks. The main objective of this research is to provide virtual learning environments (VLEs) with a framework able to recognize the student's motivation. The inference machine considers the personality traits, motivational factors obtained through the behavioral patterns, and the text subjectivity available in the communication tools of VLEs.

Keywords. Affective student modeling, bayesian virtual learning environment

\section{Introdução}

Os resultados obtidos pela Neurociência e Ciências Cognitivas, em conjunto com a Ciência da Computação, apontam para a compreensão não apenas de como os processos cognitivos acontecem, mas também como, nestes últimos, se estabelece a função da afetividade (Damásio, 1996; Ortony, Clore e Collins, 1999; Scherer, 2005). Associada à Computação Afetiva (Picard, 1997), tal compreensão vem ensejando pesquisas e o desenvolvimento de ambientes computacionais direcionados ao ensino e aprendizagem.

A Computação Afetiva congrega técnicas da Inteligência Artificial (IA), Engenharia de Software e Engenharia da Computação, objetivando desenvolver tecnologias e teorias com o intuito de entender a afetividade e seu papel nas experiências humanas. Contudo, persistem dificuldades para modelar as emoções em sistemas computacionais, basicamente devidas a três fatores, segundo Bercht (2007): ao 
pensamento cartesiano existente até recentemente nas ciências, que separa a razão da emoção; ao problema de definição dos termos associados à afetividade; e, por fim, de ordem computacional, ao uso de lógicas e formalismos voltados à representação do conhecimento.

O problema da dicotomia entre cognição e afetividade perdurou por muitos séculos, desde que Platão dividiu o corpo da "alma" estruturando-a na tríade "cognição", "emoção" e "motivação". Com o advento dos computadores, os cientistas da cognição passaram a recorrer à nova tecnologia como instrumento para representar e simular processos mentais. A analogia entre o computador e o cérebro humano, em que ambos possuem um aparato físico (eletrônico e biológico-neuronal, respectivamente) e virtual (conhecimentos, capacidade de cálculo, inferências), influenciou a adoção da hipótese de que a mente nada mais é que um mecanismo de processamento de informações e o corpo seu suporte e condição para seu funcionamento. A ênfase na abordagem de processamento de informações (codificação, armazenagem e recuperação) resultou em que os pesquisadores das ciências cognitivas não privilegiassem as relações entre a cognição e os aspectos afetivos (Eysenck e Keane, 1994).

Nos anos 60, $\operatorname{Simon}^{1}(1967)$, um dos precursores da IA, reconheceria a importância de dotar as teorias do processamento das informações cognitivas com aspectos de motivação e de emoção. Embora pesquisadores de diversas áreas (Piaget, 2005; Vygotsky, 2001) demonstrassem interesse nessa relação, somente a partir da década de 80 a afetividade nas atividades cognitivas passa a ser especialmente enfatizada nas teorias de Zajonc (1980) e Lazarus (1982). Mais tarde, nos anos 90, as pesquisas dos neurocientistas Damásio (1996) e Le Doux (2001), publicadas em revistas importantes, mostraram evidências das relações entre cognição e afetividade.

Quanto ao problema da definição, os termos afetividade, emoção, estado de ânimo/humor, motivação, sentimento, traços de personalidade, etc. são abordados por várias teorias psicológicas e, mesmo dentro delas, segundo diferentes visões. Por isso, as maneiras distintas de definir os fenômenos afetivos geram confusão na diferenciação de cada um deles. Scherer (2005) apresentou critérios para diferenciar os fenômenos afetivos, a partir dos quais serão definidos os termos relevantes para efeito deste trabalho.

Afetividade consiste na habilidade humana para experimentar sentimentos positivos ou negativos, e a eles reagir. É um termo genérico que engloba todos os fenômenos afetivos associados à dimensão afetiva. Emoção, embora no senso comum seja compreendida como sinônimo de afetividade e muitas vezes confundida com sentimento, refere-se a um estado afetivo breve, de alta intensidade e de resposta sincronizada a um evento. Uma emoção pode ser classificada em primária (básica ou utilitária) e secundária (ou social). As emoções primárias (medo, raiva, tristeza, alegria, surpresa, desprezo e aversão) servem para garantir a sobrevivência humana e foram amplamente discutidas por Ekman (1999) em pesquisas realizadas a partir de diferentes culturas. Já as emoções secundárias (arrogância, preocupação, inquietação, mágoa, entusiasmo, espanto, repulsa, etc.) são adquiridas ou aprendidas a partir das primárias, conforme se vivencia uma série de situações cotidianas e suas consequências. Estados de ânimo, uma classe de fenômenos afetivos e noção fundamental neste trabalho, 
representam um tipo de emoções de segundo plano (Damásio, 1996), de caráter ondulatório e difuso, de baixa intensidade, de longa duração, podendo ser originados a partir das emoções primárias e secundárias (Rosenberg, 1998).

A dificuldade na modelagem dos fenômenos afetivos em sistemas computacionais está no uso de modelos criados sob o método científico cartesiano, em que os mecanismos cognitivos são estudados através da redução (reducionismo - o mundo complexo deve ser dividido em partes mais simples.). Esses modelos formais tratam do conhecimento incerto e de raciocínio, da aprendizagem a partir das observações, da comunicação, da percepção e das ações a serem tomadas, embasados na resolução de problemas de maneira procedimental não trazendo à luz a afetividade.

Os formalismos podem ser divididos em duas grandes abordagens (Sternberg, 2000): simbólica ou distribuída. A representação simbólica reúne as formas declarativa (saber o quê) e não-declarativa ou procedural (saber como). O conhecimento pode ser expresso na forma de palavras ou símbolos, onde o conceito - ideia sobre algo - é unidade fundamental. Os métodos formais dessa abordagem são: a lógica BDI (Jaques, Lehmann e Pesty, 2009); a rede bayesiana (e extensões) (Pantarolo, 2008); a lógica fuzzy (Bica e Verdin, 2008); e os processos de decisão de Markov (Bui et al., 2008). Por outro lado, a representação distribuída engloba a abordagem conexionista, onde os modelos são compostos por redes automáticas que simulam o comportamento dos neurônios humanos (redes neuronais).

Fellous, Armony e Le Doux (2002) afirmam que as técnicas de IA simbólica podem ser utilizadas para modelar os processos cognitivos ${ }^{2}$ dos fenômenos afetivos, embora cada um deles tragam dificuldades teóricas que devem ser consideradas. $\mathrm{O}$ uso de lógica BDI (Beliefs, Desires e Intention) é interessante para explicar e sintentizar o comportamento afetivo a partir das atitudes mentais como crenças (Beliefs), desejos (Desires) e intenções (Intention). Por outro lado, a lógica fuzzy modela informações consideradas vagas, descritas principalmente em linguagem natural ou obtidas por sensores em tempo-real; e os processos de decisão de Markov, que resolvem problemas de decisão sequiencial ou a modelagem de transição de um processo para outro. Por fim, a rede bayesiana parte para a descrição do problema na forma qualitativa, por meio da representação de uma rede semântica, e quantitativa, cujo raciocínio é baseado de acordo com as leis de teoria da probabilidade. Esse modelo tem sido largamente utilizado para modelar domínios de conhecimento incerto e complexos como os que tratam dos aspectos afetivos.

Em termos computacionais, ou da IA, para modelar fenômenos afetivos é preciso considerar um dos modelos para a representação de conhecimento e identificar as tecnologias que o suportam (Gratch e Marsella, 2004). Contudo, não há modelos perfeitos e capazes de predizer os estados afetivos. Cada modelo tem seu lado positivo e negativo e, algumas vezes, combinar modelos pode render melhores resultados do que a aplicação de um único (Picard, 1997).

Vicari (2007) destaca os fatores que devem ser considerados na escolha de um formalismo aplicável a determinado domínio de estudo: 1) a natureza do problema; 2) o tratamento da incerteza; 3) a estruturação do domínio; e 4) a eficiência dos algoritmos. A autora também considera a possibilidade de uso de formalismos híbridos, o que pode 
representar mais precisamente os problemas de domínio e de integração dos mecanismos de raciocínio, planejamento e aprendizagem, de modo a tornar o sistema mais flexível, principalmente em domínios dinâmicos como os relacionados à afetividade.

Este artigo apresenta estudos e a avaliação de resultados obtidos na aplicação de uma rede bayesiana na inferência dos estados de ânimo durante a aplicação de objeto de aprendizagem em alunos de nível técnico nos semestre 2009/1 e 2010/1. Na próxima seção, apresentam-se os fundamentos de uma rede bayesiana. Na seção 3, faz-se uma compilação dos trabalhos da literatura relacionados aos métodos de inferência baseados em redes bayesianas. $\mathrm{Na}$ seção 4 apresenta-se o ROODA, ambiente virtual de aprendizagem de aplicação deste estudo. A seção 5 trata do modelo afetivo de aluno e a seção 6, a máquina de inferência proposta. Finalmente, são feitas algumas considerações a propósito do trabalho desenvolvido.

\section{Redes bayesianas}

Uma rede bayesiana $(\mathrm{RB})$ é um tipo de rede semântica representada por um grafo orientado acíclico, em que cada nodo (ou nó) é identificado com informações de probabilidade (Russel e Norving, 2004). Os nodos representam as variáveis aleatórias (discretas ou contínuas) do problema com as medidas de incerteza associadas. As ligações entre os nodos são feitas por arcos que definem os vínculos. Os arcos identificam a precedência lógica ou influência causal entre as variáveis conectadas. Essa precedência, ou influência, determina a distribuição da probabilidade condicional, ou seja, é feita uma quantificação do efeito dos nodos pais sobre os filhos.

A RB pode ser analisada sob duas perspectivas: qualitativa, pois o modelo gráfico representa a dependência entre os nodos, e quantitativa, por meio de tabelas de probabilidades condicionais (TPC). Assim, os motivos que justificam a opção por uma RB neste trabalho são: 1) a RB permite expressar as assertivas de independência de forma visual e, por isso, pode-se ter uma visão semântica do problema; 2) a RB é apropriada para representar e raciocinar com a incerteza, com probabilidades e imprecisão, características importantes que envolvem o tratamento dos aspectos afetivos; 3) a RB representa e armazena uma distribuição conjunta de forma reduzida, explorando a esparsidade do relacionamento entre as variáveis; e 3) a RB torna o processo de inferência eficiente do ponto de vista computacional, embora a distribuição de probabilidade possa crescer exponencialmente.

Assim, representa-se o modelo do aluno em uma RB através das relações das variáveis representadas pelos traços de personalidade, pelos fatores motivacionais obtidos a partir dos padrões comportamentais e pela subjetividade afetiva reconhecida nos relatos impressos.

\section{Trabalhos relacionados}

As RBs são largamente aplicadas em sistemas especialistas ou sistemas baseados em conhecimento (Flores, 2002), construídos para um determinado domínio (diagnóstico médico, por exemplo). Na última década, têm se destacado na representação da avaliação cognitiva dos aspectos afetivos, principalmente a partir das 
ações e outras informações (por exemplo, expressão facial) dos usuários em ambientes computacionais.

Pesquisadores da Microsoft (Breese e Ball, 2000) investigaram o uso de RBs na modelagem das emoções e personalidade do usuário. Informações referentes às expressões faciais e de fala eram evidências para que um agente conversacional inferisse a emoção e personalidade do usuário, e gerasse comportamentos adequados para com ele interagir. A mesma abordagem foi adotada por Kshirasagar e Magnenat-Thalmann (2002), mas com a inclusão da inferência dos estados de ânimo (bom e ruim).

Encontram-se, na literatura, trabalhos que propõem um modelo bayesiano para prever o estado emocional do usuário. Xiang et alli (2006) apresentam a Rede de Transição de Estado Mental, que tem por objetivo calcular a probabilidade de ocorrência de uma nova emoção em função das condições atuais do ambiente. Tais condições influenciam a emoção corrente, provocando uma transição para uma nova.

Outros autores propõem o uso de RBs em agentes computacionais que inferem as emoções de alunos em jogos colaborativos e sistemas educacionais. $\mathrm{O}$ agente social proposto por Boff (2008) leva em consideração as características individuais do aluno como desempenho, perfil social, aceitação (por parte de colega), estado afetivo, traços de personalidade, entre outros para inferir o perfil do aluno na proposição de grupos de trabalhos. O modelo de Conati (2009) prevê os estados emocionais através de uma avaliação das interações do aluno no jogo a partir dos objetivos e preferências (a expressividade corporal também é evidência). O modelo desenvolvido trata da inferência das emoções em relação a si próprio e em relação a um agente pedagógico que o apóia durante as atividades do jogo.

Abordagem semelhante foi adotada por Pantarolo (2008). Entretanto, a principal contribuição do autor está na inferência das emoções do aluno em relação a si e aos demais colegas com os quais interage no jogo colaborativo. $\mathrm{O}$ modelo afetivo, desenvolvido por Pantarolo considera a dinâmica do jogo, isto é, as ações coordenadas e simétricas por parte dos alunos-jogadores para solucionar conjuntamente os desafios do jogo.

Este trabalho tem forte influência dos trabalhos de Pantarolo (2008), Boff (2008) e Conati (2009) no que se refere à construção e utilização de redes bayesianas na avaliação cognitiva dos estados de ânimo.

\section{Ambientes virtuais de aprendizagem}

O ROODA $^{3}$ (Rede cOOperativa De Aprendizagem), institucionalizado pela UFRGS em 2005 , é um ambiente virtual de aprendizagem ${ }^{4}$ desenvolvido com base em princípios construtivistas, tendo implícita a concepção epistemológica interacionista (Piaget, 1973). As funcionalidades disponíveis no ROODA podem ser agrupadas nas classes: Recursos Gerais, Acompanhamento de Atividades, Publicação de Materiais e Comunicação.

O grupo de funcionalidades que contém Recursos Gerais permite verificar e alterar dados cadastrais (Dados pessoais), personalizar a interface (Configurações), registrar compromissos (Compromissos) com a disciplina, verificar o histórico de tempo 
e número de acessos às funcionalidades (InterROODA) e acessar o item Ajuda para os problemas com o ambiente. As funcionalidades de Acompanhamento de Atividades são responsáveis pela disponibilização dos conteúdos (Aulas e Biblioteca), dos exercícios (Exercícios) e da participação de enquetes (Enquete). Já os recursos Webfólio pessoal e de grupo constituem as funcionalidades de Publicação de Materiais. Por fim, as ferramentas de comunicação síncrona (Bate-papo e A2) e assíncrona (Fórum, Diário de Bordo, Lista de Discussão e Contatos) integram o grupo de funcionalidades de Comunicação. Essas possibilitam encontros virtuais e espaços de convivência, sustentando o movimento de negociações, discussões e coordenações durante a realização da disciplina.

A máquina de inferência dos estados de ânimo será acoplada ao AVA ROODA através da funcionalidade ROODAafeto, ora em desenvolvimento pela equipe do NUTED/UFRGS. A nova funcionalidade integrará a classe Recursos Gerais do AVA ROODA.

\section{O modelo afetivo do aluno}

A proposta de modelo afetivo do aluno, representado através de uma rede semântica, ilustrada na Figura 1, baseia-se no modelo de Scherer (Scherer, 2005).

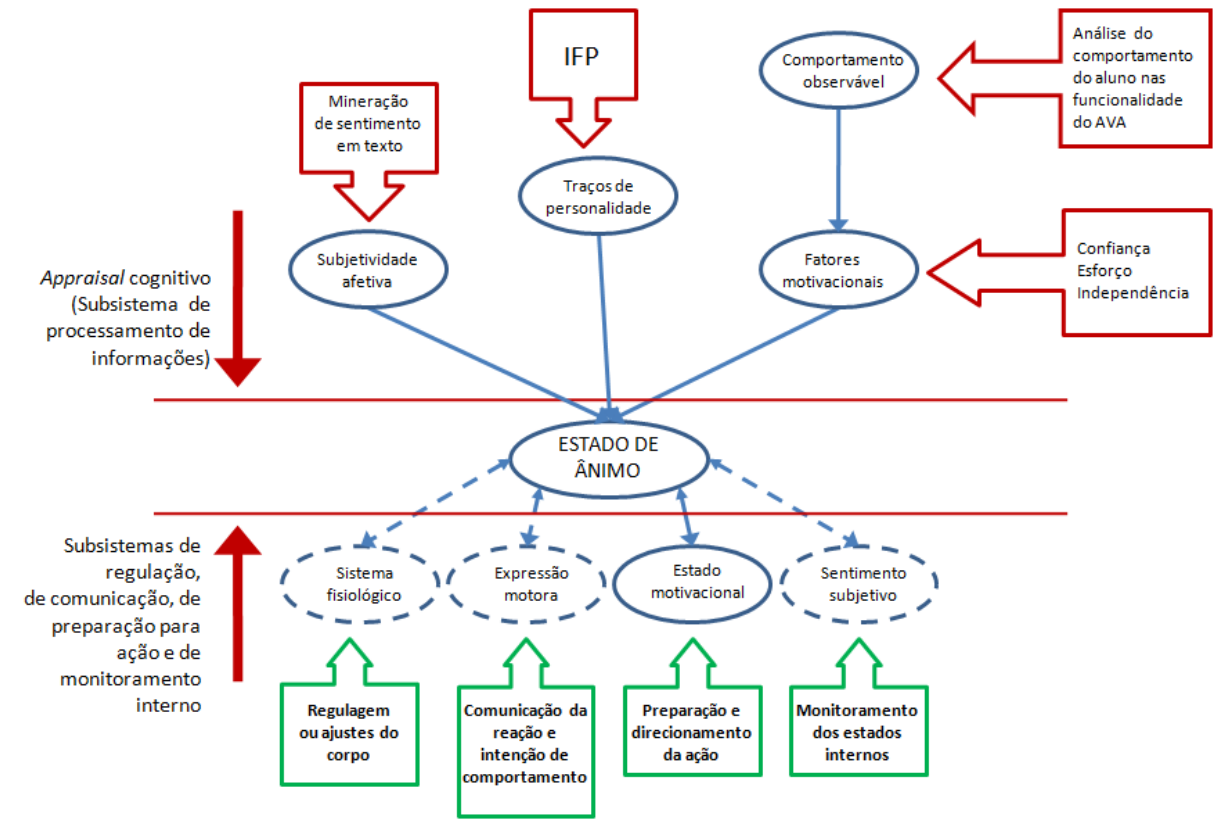

Figura 1. Classe de variáveis e relações do modelo afetivo do aluno

Neste modelo, a subjetividade afetiva em texto é considerada uma das variáveis de influência ao estado de ânimo do aluno. A subjetividade afetiva é entendida como o julgamento unilateral (Dorsch, Häcker e Stapf, 2008). Tal julgamento é considerado a partir do ponto de vista do aluno, quando este registra preocupações, sucessos e motivações nos textos disponibilizados nas funcionalidades de comunicação síncrona e assíncrona do AVA. A subjetividade afetiva é inferida pelo framework AWM (Affective Word Mining), o qual tem por objetivo classificar as palavras de conotação afetiva de um texto. O processo de mineração extrai, em cada frase da mensagem, os lexemas 
afetivos, classificados no estado de ânimo mais apropriado, conforme descrito em (Longhi et al., 2009).

Os aspectos comportamentais são entendidos como um conjunto constante de ações adotadas pelo aluno no AVA. Dessa forma, os padrões de comportamento sinalizam o grau motivacional (Confiança, Esforço e Independência), que pode ser um indicador dos estados de ânimo. Cada fator motivacional é obtido através de variáveis relacionadas ao comportamento observável, tais como: número de acessos (NA) a uma funcionalidade, número de contribuições $(\mathrm{NC})$ em fóruns, freqüência (FP) e modo de participação (MP), pedidos ou prestação de ajuda (PA), número de visitas (NV) e tempo de permanência (TP) na sessão. O grau motivacional é obtido pelo framework BFC (Behavioral Factor Calculation) descrito em (Longhi, Behar e Bercht, 2010).

Os traços de personalidade constituem padrões através dos quais o sujeito percebe a realidade, e explicam como se relaciona com os demais. Normalmente, são determinados através de modelos caracterizados como fatores (ou dimensões), que denotam a especificidade de um indivíduo. Estes fatores são obtidos através da aplicação de testes psicométricos. Na pesquisa em curso utiliza-se o instrumento IFP (Inventário Fatorial da Personalidade) elaborado por Pasquali, Azevedo e Ghesti (1997), com aplicação e análise pela psicóloga Daniela Forgiarini Pereira ${ }^{5}$ a fim de compreender essas relações. A escolha do IFP se justifica pelo fato de representar um instrumento adaptado à realidade brasileira, fidedigno e de natureza verbal mais consistente com os propósitos da pesquisa. A classe traços de personalidade envolve 14 variáveis aleatórias ${ }^{6}$, a saber: assistência, intracepção, afago, deferência, afiliação, dominância, denegação, desempenho, exibição, agressão, ordem, persistência, mudança e autonomia.

\section{Máquina de inferência}

A tecnologia de RB é a abstração computacional escolhida para apropriar a rede semântica descrita na seção anterior. Tal escolha se deve ao fato de representar uma tecnologia adequada por considerar os aspectos de incerteza inerentes à dimensão afetiva. E também por tratar os aspectos dinâmicos do processo de inferência (o modelo afetivo é constantemente atualizado conforme os dados estejam sendo capturados).

Entende-se que reunir os traços de personalidade a fatores motivacionais (FM) fornece condições para se poder responder à questão sobre qual é o possível estado de ânimo do aluno, em uma disciplina/turma desenvolvida (em parte ou toda) através de um AVA. A subjetividade afetiva em texto é considerada para atribuir maior ou menor peso na inferência, por ser manifestação espontânea do aluno, quando o fator desejabilidade social $^{7}$ não for relevante. Aplica-se o modelo afetivo de aluno sobre uma $\mathrm{RB}$ de forma a se obter inferências estatísticas a partir das probabilidades associada aos eventos observados.

A RB apresentada na Figura 2, conhecida por MIM (Mood Inference Machine), suporta as relações das variáveis visualizadas na Figura 1. As variáveis independentes podem assumir os seguintes estados e valores: 1) traços de personalidade (assistência (Ass), intracepção (I), afago (Af), deferência (Def), afiliação (Afi), dominância (Do), denegação (Den), desempenho (Des), exibição (Ex), agressão (Ag), ordem (O), 
persistência $(\mathrm{P})$, mudança $(\mathrm{M})$ e autonomia (Aut)): valores muito alto $(>70)$, alto (entre 60 e 70), equilíbrio (entre 40 e 60 ), baixo (entre 30 e 40) e muito baixo $(<30) ; 2$ ) confiança $(\mathrm{C})$, esforço (E) e independência (I): muito alto, alto, médio, baixo e muito baixo; e 3) subjetividade em texto (S): animado, desanimado, satisfeito, insatisfeito.

Uma vez especificada a topologia da rede, foram definidas as tabelas de probabilidade condicional (TPC) de cada nodo. Geralmente, os valores de probabilidade de cada TPC são obtidos através de análises estatísticas em um grande volume de dados. Para a pesquisa em curso optou-se por inicializar as TPCs através de valores obtidos a partir de estudos estatísticos de 184 alunos em nível de graduação e de nível técnico. Desses, apenas 147 foram considerados, já que os demais apresentaram fator de desejabilidade social muito elevado ou, então, incorreram em contradição durante a aplicação do IFP.

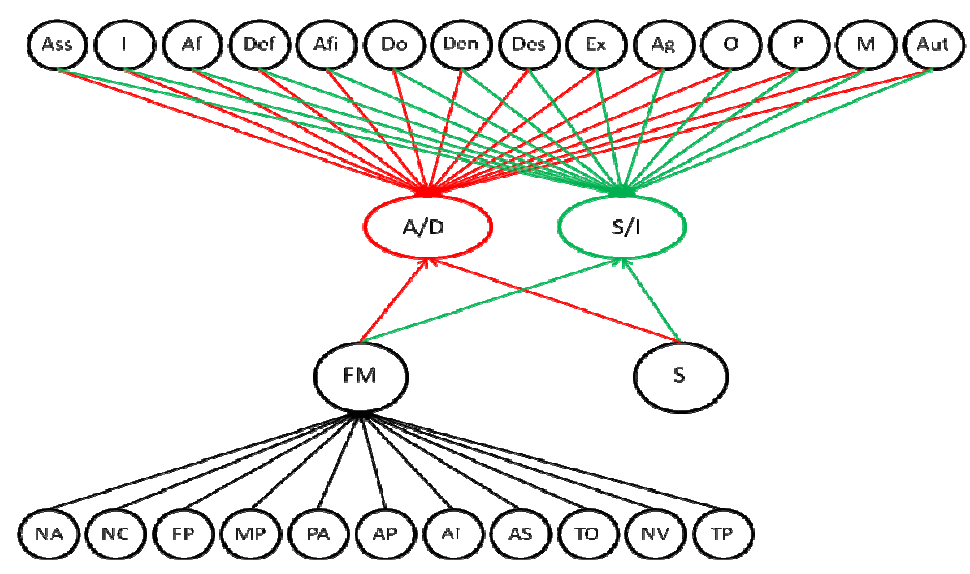

Figura 2. Modelo de rede bayesiana para inferência dos estados de ânimo

Inicialmente, os valores de probabilidade dos nodos (ou variáveis) independentes possuem valores iguais (20\% para os valores de traços de personalidade e fatores motivacionais e $25 \%$ para os de subjetividade em texto). Os valores são atualizados conforme a máquina for recebendo informações sobre os dados do aluno. Esse ajuste caracteriza as novas observações realizadas sobre o aluno.

Para determinar, por exemplo, o grau do fator motivacional (FM), a máquina de inferência deve calcular a probabilidade para a confiança: $p(\mathrm{FM}=\mathrm{C})$, tal que, $p(\mathrm{FM}=\mathrm{C} / \mathrm{NA}, \mathrm{NC}, \mathrm{FP}, \mathrm{MP}, \mathrm{PA}, \mathrm{AP}, \mathrm{AT}, \mathrm{AS}, \mathrm{TO}, \mathrm{NV}, \mathrm{TP})$; para o esforço: $p(\mathrm{FM}=\mathrm{E})$, tal que, $p(\mathrm{FM}=\mathrm{E} / \mathrm{NA}, \mathrm{NC}, \mathrm{FP}, \mathrm{MP}, \mathrm{PA}, \mathrm{AP}, \mathrm{AT}, \mathrm{AS}, \mathrm{TO}, \mathrm{NV}, \mathrm{TP})$; e para a independência: $p(\mathrm{FM}=\mathrm{I})$, tal que, $p(\mathrm{FM}=\mathrm{I} / \mathrm{NA}, \mathrm{NC}, \mathrm{FP}, \mathrm{MP}, \mathrm{PA}, \mathrm{AP}, \mathrm{AT}, \mathrm{AS}, \mathrm{TO}, \mathrm{NV}$, $\mathrm{TP})$. Embora o cálculo pareça simples adquire complexidade elevada em face do crescimento exponencial das TPCs.

\section{Considerações finais}

O reconhecimento dos estados afetivos envolve a captura e a análise dos sinais, os quais podem ser transformados em dados com vista à construção do modelo afetivo do usuário. Através dele, o sistema computacional pode inferir as razões pelas quais o usuário experimentou determinado episódio afetivo. Para isso, o sistema computacional necessita construir e atualizar dinamicamente o modelo afetivo-cognitivo do usuário, 
contemplando a incerteza dos dados obtidos no reconhecimento e a influência do cognitivo no afetivo, e vice-versa.

O presente trabalho apresenta o estado da arte do processo de construção de máquina de inferência dos estados de ânimo de aluno a ser inserida na funcionalidade ROODAafeto do AVA ROODA. Para avaliar a possibilidade de construção, foi conduzido um experimento, cujos dados foram submetidos a um protótipo de rede implementada através do software Hugin Lite (v.7.3) ${ }^{8}$.

Na simulação, o aluno conta com uma representação cognitiva e afetiva limitada às características indispensáveis aos propósitos da pesquisa. Esse modelo tem o intuito de formalizar suas inter-relações, seja com o sistema, seja com os colegas, seja ainda com os professores e/ou tutores. A abordagem dinâmica pressupõe: a) as incertezas advindas do tipo de modelo a ser empregado para representar a afetividade (modelo de Scherer); e b) eventuais falhas de compreensão das variáveis utilizadas, dada a provável precariedade dos "sinais" capturados. O objetivo é construir um sistema computacional adaptativo, isto é, suficientemente dinâmico e flexível para as características individuais do aluno.

\footnotetext{
Notas

${ }^{1}$ Hebert Simon foi agraciado com o prêmio Turing Award em 1975 por suas contribuições à IA, e em 1978, com o Prêmio Nobel de Economia pela pesquisa precursora sobre o processo de tomada de decisões dentro de organizações econômicas.

2 Os autores da teoria cognitivista (Scherer, 2005; Lazarus, 1982) acreditam que o indivíduo faz constantes avaliações cognitivas de uma situação. O produto dessa análise determina as modificações fisiológicas do corpo, o tipo de ação a ser tomada, a expressividade motora e o sentimento subjacente.

${ }^{3}$ Disponível em HTTP://www.ead.ufrgs.br/rooda

${ }^{4}$ Um ambiente virtual de aprendizagem (AVA) pode ser definido como um espaço virtual formado pelos sujeitos participantes (aluno, professor e tutor) e materiais de ensino e de aprendizagem nele contidos; pelas interações que ocorrem e relações que se formam; e pelas formas de comunicação disponibilizadas para as trocas de informações. Esse espaço virtual é muitas vezes conhecido sob a designação de plataforma (Behar, 2009).

${ }^{5}$ Mestre em Psicologia pela UFRGS, CRPRS 07/12056, doutoranda no Programa de Pós-graduação em Informática na Educação (UFRGS).

${ }^{6}$ O IFP trata 15 variáveis e mais duas de controle (desejabilidade social e mentira). A variável heterosexualidade não será utilizada na pesquisa.

7 Característica da pessoa com propensão para transmitir uma imagem culturalmente aceitável. Eventualmente, pode ocasionar um viés na investigação científica. Este fator é obtido através do IFP.

${ }^{8}$ A rede apresentada na Figura 2 sofreu ajustes em função das limitações do aplicativo.
}

\section{Referências Bibliográficas}

BALL, G.; BREESE, J. Relating Personality and Behavior: Posture and Gestures. In: PAIVA, A. M. (Ed.): Affective Interactions, LNAI 1814, 2000, p. 196-203.

BEHAR, P. A.. Modelos Pedagógicos em Educação a Distância. Porto Alegre: Artmed, 2009.

BERCHT, M. Computação Afetiva: vínculos com a psicologia e aplicações na educação. In: Psicologia \& Informática: produções do III PsicoInfo e II Jornada do NPDI. São Paulo: Conselho Regional de Psicologia de São Paulo. 2007. p.106-115.

BICA, F.; \& VERDIN, R. InteliWeb: The E-Learning System that Recognizes Aspects of SelfEfficacy. In: VICCARI, R. M.; JAQUES, P. J.; e VERDIN, R. (Org.). Agent-based 
tutoring systems by cognitive and affective modeling, Hershey, PA: Information Science Publishing (IGI Global). p.156-177. 2008.

BOFF, E. Colaboração em ambientes inteligentes de aprendizagem mediada por um agente social probabilístico. 2008. 163 f. Tese (Doutorado: Programa de Pós Graduação em Computação), Instituto de Informática, Universidade Federal do Rio Grande do Sul, Porto Alegre, 2008.

CONATI, C.; MACLAREN, H. 2009. Empirically building and evaluating a probabilistic model of user affect. In: User Modeling and User-Adapted Interaction, v.19, n.3, Jan. 2009, p. 267-303.

DAMÁSIO, A. O Erro de Descartes: emoção, razão e o cérebro humano. Tradução: Dora Vicente e Georgina Segurado. São Paulo: Cia das Letras, 1996.

DORSCH, F.; HÄCKER, H.; STAPF, K.H. Dicionário de psicologia Dorsch. LEÃO, E.C. (Trad.). Petrópolis, RJ: Vozes, 2008.

EKMAN, P. Basic Emotions. In: DALGLEISH, T.; POWER, T. (Eds.). The Handbook of Cognition and Emotion. Sussex, U.K.: John Wiley \& Sons, Ltd. 1999. p. 45-60.

EYSENCK, M. W. \& KEANE, M. T. Psicologia Cognitiva: um manual introdutório. Trad.: Wagner Gesser e Maria Helena Fenalti Gesser. Porto Alegre: Artes Médicas, 1994.

FELLOUS J. M.; ARMONY J. L.; LE DOUX J. E.. Emotional Circuits and Computational Neuroscience. In: ARBIB, M.A. (Ed.), The handbook of brain theory and neural networks, Second Edition. Cambridge, MA: The MIT Press. Nov, 2002.

FLORES, C. D. Fundamentos dos Sistemas Especialistas. In: BARONE, D. A. C. (Org.). Sociedades Artificiais: A Nova Fronteira da Inteligência nas Máquinas, Porto Alegre: Bookman (ArtMed), 2002, p. 127-154.

GRATCH, J., MARSELLA, S.: A Domain-independent framework for modeling emotion. Journal of Cognitive Systems Research, v.5, n.4, p. 269-306. 2004.

KSHIRSAGAR, S. \& MAGNENAT-THALMANN, N. A Multilayer Personality Model. In: $2^{\text {nd }}$ INTERNATIONAL SYMPOSIUM ON SMART GRAPHICS, 2002, Hawthorne, NY, USA. Proceedings... New York: ACM Press, 2002. p.107-115.

LAZARUS, R. S. Thoughts on the relations between emotion and cognition. In: American Psychologist, v.37, n.9, p. 1019-1010, Sep. 1982.

LE DOUX, J. O cérebro emocional: os misteriosos alicerces da vida emocional. SANTOS, T. B. (Trad.). Rio de Janeiro: Objetiva, 2001.

LONGHI, M. T.; BEHAR, P. A.; BERCHT, M. e SIMONATO, G. Investigando a subjetividade afetiva na comunicação assíncrona de ambientes virtuais de aprendizagem. In: XX SIMPÓSIO BRASILEIRO DE INFORMÁTICA NA EDUCAÇÃO, 2009, Florianópolis, SC. Anais do SBIE2009... Nov. 2009.

LONGHI, M. T.; BEHAR, P. A.; BERCHT, M. e SIMONATO, G. Os fatores motivacionais e os estados de ânimo em ambientes virtuais de aprendizagem. Artigo submetido ao IE2010, Chile. 2010.

ORTONY, A; CLORE, G.; COLLINS. A. The cognitive structure of emotions. Cambridge: Cambridge University Press, 1988.

PANTAROLO, E. Modelagem probabilistic de aspectos afetivos do aluno em um jogo educacional colaborativo. PGIE/UFRGS. Tese de doutorado, 2008.

PASQUALI, L.; AZEVEDO, M. M.; GHESTI, I. Inventário Fatorial de Personalidade: manual técnico e de aplicação. São Paulo: Casa do Psicólogo. 1997.

PIAGET, J. Inteligencia y afectividad. Buenos Aires: Aique, 2005.

PIAGET, J. Estudos Sociológicos. Rio de Janeiro: Forense. 1973.

PICARD, R. W. Affective Computing. Cambridge: MIT Press. 1997.

ROSENBERG, E.L. Levels of analysis and the organization of affect. Review of General Psychology, v. 2, n. 3, p. 247-270. 1998.

RUSSELL, S.J.; NORVING, P. Inteligência Artificial. Rio de Janeiro: Elsevier, 2004.

V. $8 \mathrm{~N}^{\mathrm{o}}$ 3, dezembro, 2010 
SCHERER, K. R. What are emotions? And how can they be measured? In: Social Science Information, v.44, n.4, 2005, p. 695-729.

SIMON, H. Motivational and Emotional Controls of Cognition. Psychological Review, v. 74, n. 1, p.29-39, Jan. 1967.

STERNBERG, R. J.. Psicologia cognitiva. OSÓRIO, M. R. B.(Trad.). Porto Alegre: Artes Médicas Sul, 2000.

VICARI, R. M. As grandes linhas da Inteligência Artificial. Notas de aula. 2007.

VYGOTSKI, L. S. A construção do pensamento e da linguagem. São Paulo: Martins Fontes, 2001.

XIANG, H; XIAO, S.; REN, F.; KUROIWA, S.. A mind model for an affective computer. International Journal of Computer Science and Network Security (IJCSNS), v. 6, n. 6, Jun. 2006

ZAJONC, R. B. Feeling and thinking: preferences need no inferences. American Psychologist, v.35, n.2, p. 151-175, Feb. 1980. 